ORIGINAL ARTICLE

\title{
Glucose-6-phosphate dehydrogenase activity in male premature and term neonates
}

\author{
O Mesner, C Hammerman, D Goldschmidt, B Rudensky, D Bader, M Kaplan
}

Arch Dis Child Fetal Neonatal Ed 2004;89:F555-F557. doi: 10.1136/adc.2004.049148

See end of article for authors' affiliations .......................

Correspondence to: Dr Kaplan, Department of Neonatology, Shaare Zedek Medical Center, PO Box 3235, Jerusalem 91031, Israel; kaplan@cc. huji.ac.il

Accepted 7 May 2004
Background: Glucose-6-phosphate dehydrogenase (G6PD) activity is higher in term neonates than in adults. Some studies have suggested that activity may be even higher in preterm infants.

Objectives: To determine if G6PD activity is higher in preterm than term neonates, and whether higher activity would interfere with diagnosis of G6PD deficiency in premature infants.

Methods: G6PD activity was determined in the first 48 hours after delivery in male premature, term, and near term infants. G6PD deficient neonates were separated, and the remaining premature infants compared with healthy, male, G6PD normal, near term and term neonates.

Results: Ninety four premature infants (mean (SD) gestational age 31.9 (3.8) weeks (range 23-36)) were studied. In four, G6PD activity was 0.8-1.8 U/g haemoglobin ( $\mathrm{Hb})$, which is clearly in the deficient range with no overlap into the normal range. G6PD activity in the remaining premature infants was significantly higher than in 24 near term and term neonates (gestational age $\geqslant 37$ weeks) (14.2 (4.6) v 12.0 (3.8) U/g $\mathrm{Hb})$. Further analysis showed that significance was limited to those born between 29 and 32 weeks gestation, in which group G6PD activity was significantly higher than in those born before 29 weeks gestation, at 33-36 weeks gestation, and $\geqslant 37$ weeks gestation.

Conclusions: G6PD activity is higher in premature infants born between 29 and 32 weeks gestation than in term neonates. This did not interfere with diagnosis of G6PD deficiency.
G lucose-6-phosphate dehydrogenase (G6PD) deficiency is a common defect, affecting hundreds of millions of people, with a worldwide distribution. ${ }^{1}$ Its incidence varies from $<3 \%$ in the United States and Europe to $25 \%$ in some parts of Africa and the Middle East. Within any given area, the incidence may vary between population subgroups. ${ }^{2}$ In Israel, in some subsets of Sephardic Jews there is a high incidence of the G6PD Mediterranean mutation. ${ }^{3}{ }^{4}$

Although best known for acute, life threatening haemolytic crises (favism), G6PD deficiency is also associated with severe neonatal jaundice, with the potential of bilirubin encephalopathy, or kernicterus, if untreated. ${ }^{56}$ In a recent report of the informal United States based Kernicterus Registry, at least $21 \%$ of readmitted infants with kernicterus had documented G6PD deficiency. ${ }^{7}$ Premature neonates are susceptible to kernicterus at lower concentrations of serum total bilirubin than are term neonates. It is therefore possible that in population groups with a high incidence of G6PD deficiency, this condition may play a role in the pathogenesis of kernicterus in premature infants. However, there are few studies of G6PD deficiency in premature infants.

Normal neonates born at term have been shown to have higher G6PD activity than adults, ${ }^{8-11}$ and some studies have suggested even higher activity in premature infants. ${ }^{12-15}$ We therefore hypothesised that premature infants would have even higher G6PD activity than term neonates, and asked whether this increase would influence the ability to diagnose the deficiency state. In this study, we determined, quantitatively, normal values for red blood cell G6PD activity in male premature neonates and compared these values with those of healthy term and near term male infants.

\section{METHODS}

\section{Clinical protocol}

The study was approved by the Institutional Review Boards of both Bnai Zion and Shaare Zedek Medical Centers and further sanctioned by the Israel Government Ministry of Health.
The amount of blood required for study purposes was small and was drawn simultaneously with blood sampling for routine laboratory tests. In this study there was no randomisation of patients, clinical or therapeutic trial, additional risk to the babies or any other deviation from routine clinical management. For these reasons, the authorities gave approval to perform the study without the need for individual parental consent.

Any male, preterm infant, $\leqslant 36$ weeks gestation, admitted to the premature care nurseries of the participating centres, was eligible for the study. Neonates with major congenital anomalies, documented sepsis, intrauterine growth retardation, or grade 3 or 4 intraventricular haemorrhage apparent at the time of the G6PD testing were excluded. Neonates with respiratory distress or receiving ventilator treatment were not excluded from the study, as such a restriction would have resulted in the exclusion of almost every neonate $\leqslant 29$ weeks gestation and many of those with higher gestational ages as well. Furthermore, we are unaware of any data suggesting that mechanical ventilation for respiratory distress syndrome influences G6PD activity. Gestational age, for the purpose of the study, was defined as the number of completed weeks subsequent to the first day of the last menstrual period, validated by ultrasound examinations during the pregnancy and confirmed by physical examination after delivery. In the event of a discrepancy, or if the gestational age could not be accurately determined, that baby was excluded from analysis. In addition to the premature infants, 24 healthy term and near term male neonates (gestational ages $\geqslant 37$ weeks) were sampled for comparison. The study was limited to male infants, as G6PD deficiency is an X-linked condition and, in males, who can be either normal or deficient hemizygotes, the G6PD status can be accurately determined. In females, on the other hand, a high number of

Abbreviations: G6PD, glucose-6-phosphate dehydrogenase; $\mathrm{Hb}$, haemoglobin 
G6PD deficient heterozygotes, a form of the condition that may be difficult to diagnose, may be encountered. ${ }^{1}$

Within 48 hours of delivery, and before any blood had been administered by transfusion, $0.25-0.5 \mathrm{ml}$ blood was collected in an EDTA-containing microtainer. In those neonates who had an indwelling vascular catheter in place, this blood was collected through the catheter. In the remaining babies, blood was sampled by venepuncture, at the time of blood sampling for a routine laboratory investigation. No baby was hypoglycaemic, hypoxic, or had metabolic acidosis at the time of sampling. This blood was filtered through cellulose to remove leucocytes, ${ }^{16}$ and the filtrate stored at $-70^{\circ} \mathrm{C}$ pending laboratory analysis.

\section{Laboratory methods}

Before the G6PD assay, the haemolysate was thawed and haemoglobin $(\mathrm{Hb})$ concentration was measured by the Coulter method (Coulter T-980; Coulter Electronics, Luton, UK). G6PD activity was determined using a commercial kit (G6PD: quantitative, ultraviolet kinetic determination in blood at $340 \mathrm{~nm}$, kit no 345 UV; Sigma Diagnostics, St Louis, Missouri, USA). The principle of the test involves the oxidation of glucose 6-phosphate to 6-phosphogluconate, and the concomitant reduction of $\mathrm{NADP}^{+}$to NADPH. These reactions occur in the presence of $\mathrm{G} 6 \mathrm{PD}$, and the rate of NADPH formation, which is proportional to G6PD activity, is measured spectrophotometrically. One international unit (U) of activity is defined as the amount of G6PD activity that will convert $1 \mu \mathrm{mol}$ glucose 6-phosphate to 6-phosphogluconate per minute.

A $0.01 \mathrm{ml}$ portion of haemolysate was added to a vial containing G6PDH assay solution comprising NADP, maleimide, buffer, and stabilising agent, mixed, and left at room temperature for 5-10 minutes. G6PD substrate solution containing glucose 6-phosphate was added to the vial, and the contents transferred to a test cuvette. The cuvette was placed in a constant temperature water bath, and the absorbance at $340 \mathrm{~nm}$ read at baseline and five minutes later, versus potassium dichromate, in a narrow width spectrophotometer (Ocean Scientific Co, Garden Grove, California, USA). The rate of increase in absorbance at $340 \mathrm{~nm}$ was calculated and expressed as U/g Hb. The manufacturer's reported normal range in healthy adults is $4.6-13.5 \mathrm{U} / \mathrm{g} \mathrm{Hb}$.

\section{Sample size}

Based on an estimated 25\% increase in G6PD activity in premature infants compared with term and near term neonates, with a power of 0.8 and an alpha value of $0.05,17$ neonates would be necessary in each group. The complete range of prematurity for study purposes (23-36 weeks) was divided arbitrarily into three approximately equal groups by gestational week, and at least 17 patients sampled in each group.

\section{Data analysis}

Based on a previous study from our laboratory in term infants, a cut off point of $5.0 \mathrm{U} / \mathrm{g} \mathrm{Hb}$ enzyme activity was chosen to delineate the G6PD deficient from the G6PD normal infants. ${ }^{11}$ To facilitate determination of normal values for G6PD activity, neonates who were clearly G6PD deficient were not included in subsequent analysis. G6PD activity for the preterm infants $(\leqslant 36$ weeks gestation) were compared with those of the term and near term neonates ( $\geqslant 37$ weeks gestation) using Student's $t$ test. When it had been confirmed that G6PD activity was higher in the premature neonates than in the term and near term group, the premature neonates were stratified into subgroups at two weekly intervals of gestational age, beginning at 23 weeks, and the subgroups compared using analysis of variance, to identify the sub- groups with significantly higher G6PD activity. Data are presented as mean (SD). Significance was defined as $\mathrm{p}<0.05$.

\section{RESULTS}

A total of 118 male neonates were sampled, of whom 94 were premature (31.9 (3.8) weeks gestation (range 23-36)) and 24 were term and near term (38.8 (1.5) weeks (range 37-42)). Four premature infants (one at 24 weeks, one at 34 weeks, and two at 36 weeks gestation) had G6PD activity of $0.8-$ $1.8 \mathrm{U} / \mathrm{g} \mathrm{Hb}$, which is clearly in the deficient range with no overlap into the normal range (below). These G6PD deficient babies were excluded from further analysis.

The mean (SD) G6PD activity for the remaining premature infants was $14.2(4.6) \mathrm{U} / \mathrm{g} \mathrm{Hb}$, which was significantly higher than that of the term infants $(12.0$ (3.8) $\mathrm{U} / \mathrm{g}$ $\mathrm{Hb} ; \mathrm{p}=0.03$ ).

Analysis of variance of the subgroups based on two weekly intervals of gestational age identified significant variation $(\mathrm{p}=0.03)$. This was localised to the 29-30 week (17.0 (4.9) U/g Hb) and 31-32 week (16.3 (5.8) U/g Hb) subgroups, the values in which were both significantly higher than those of the term neonates $(p=0.01$ and $p=0.009$ respectively). The neonates in the 29-30 and 31-32 week gestational age groups were then pooled and further analysed by comparing them with neonates born before 29 weeks gestation, those born at 33-36 weeks, and the $\geqslant 37$ week group. G6PD activity in the 22 neonates born between 29 and 32 weeks gestation (16.5 (5.5) U/g Hb) was significantly higher than the pooled values for 19 neonates of $<29$ weeks (12.1 (4.7) U/g Hb; p $=0.009$ ), the 49 neonates of 3336 weeks gestation (12.9 (4.9) U/g Hb; p = 0.008), as well as the neonates $\geqslant 37$ weeks gestation $(\mathrm{p}=0.003)$ (fig $\mathrm{l})$. The pooled values of those $<29$ weeks were not significantly different from those of 33-36 weeks $(p=0.6)$. The number of neonates in the 33-36 week gestational age subgroup is larger than the remaining groups because larger premature neonates are more often encountered.

\section{DISCUSSION}

It has long been known that term neonates at birth have higher G6PD activity than adults. ${ }^{8-11}$ A few studies have suggested that this trend may extend to premature neonates. ${ }^{12-15}$ On the basis of these findings, we hypothesised that premature infants would have even higher G6PD activity than term infants. Overall, our hypothesis was correct: G6PD activity in the pooled group of premature infants was significantly higher than in the group of term neonates. Subgroup analysis showed that there was a peak at around 30 weeks gestation, neonates born at 29-32 weeks gestation having higher G6PD activities not only than their term counterparts but also than those born before 29 weeks and those born at 33-36 weeks. G6PD activity did not differ significantly between either the very premature infants or the group of 33-36 weeks gestation and the term and near term

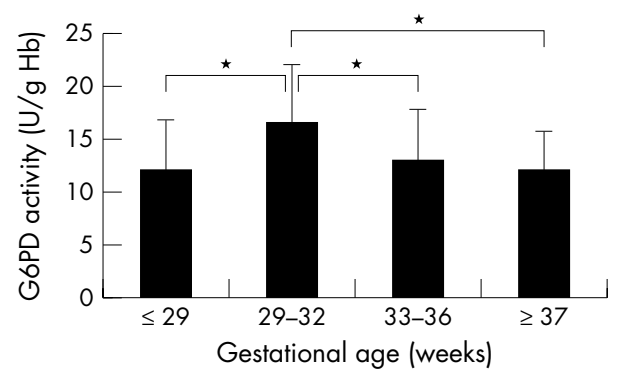

Figure 1 Glucose-6-phosphate dehydrogenase (G6PD) activity (mean (SD)) for the three subgroups of premature infants and the term and near term neonates. ${ }^{*} p<0.001$. 


\section{What is already known}

G6PD activity is higher in term neonates shortly after birth than in adults. A few studies have suggested that G6PD activity may be even higher in premature infants than in term neonates. However, these studies either have methodological faults or did not include very immature neonates in their premature patient population, and these results are therefore inconclusive.

neonates. There was no difficulty, however, in differentiating between G6PD normal and G6PD deficient neonates, as activity in the latter group was clearly lower than, and with no overlap between, that in the normal neonates.

Only a few studies of G6PD activity have been performed in premature or low birthweight neonates, and with inconsistent results. Marks and Gross ${ }^{12}$ compared premature and term newborns with adults, and found that G6PD activity in the former group was higher than in term infants of comparable age. However, their premature infant group included those aged 2 days to 1 year, and no data were available for gestational age or birth weight. Stewart and Birnbeck ${ }^{13}$ also studied premature infants, along with term neonates and adults. As in our study, they showed higher activity in premature than in term infants, and both these groups had higher activity than adults. Furthermore, decreasing gestational age correlated inversely with G6PD activity. However, in that series, no neonate of gestational age $<28$ weeks or $<1000 \mathrm{~g}$ was included. Herz et $a l^{14}$ found higher G6PD activity in infants of low birth weight than normal counterparts. However, values for infants of birth weight 865-2160 g, with gestational ages of 28-40 weeks, were pooled, with no stratification for either birth weight or gestational age. In a group of relatively large premature infants (birth weight 2141 (310) g), Oski et a ${ }^{17}$ documented that G6PD activity within the first 48 hours of life was significantly higher than in adults, but not significantly higher than in term infants.

In contrast with these studies, we prospectively studied G6PD activity in premature and term neonates enrolled shortly after birth, and found that activity in premature infants was higher than in term and near term neonates. The range of our study group extended from 23 weeks (the border of gestational viability) to 42 weeks gestation, and those born before 36 weeks stratified at two weekly intervals. Our results pinpointed the increase in G6PD activity in premature infants to the 29-32 week gestational period.

The peak G6PD activity at about 30 weeks gestation and subsequent decline in activity towards term is interesting and has not been previously described. The reason for the increase in activity is not clear. Had immaturity, or an immaturity related disease process, been a major factor in producing this phenomenon, the more immature babies would have been expected to have even higher G6PD activity than those at 2932 weeks gestation. It was previously thought that the higher G6PD activity in newborns, compared with adults, was due, in part, to the large number of immature red blood cells and reticulocytes with higher G6PD activities, in this population subgroup. However, Konrad et al corrected for reticulocyte count, and showed that a high number of these cells was not a prerequisite for the increased G6PD activity in newborn blood. Mohrenweiser et $\mathrm{al}^{15}$ did find that newborns had higher G6PD activity than adults, although no significant differences were noted when blood with a high reticulocyte count was compared with samples with normal reticulocyte values. Additional as yet undetermined factors appear to be responsible for the intrinsically higher G6PD activity in neonates and premature infants. The peak we have described suggests a maturational

\section{What this study adds}

This study supplies data on G6PD activity in male premature infants from the border of viability (23-24 weeks gestation) until 36 weeks gestation, and compares values with those of male term and near term neonates $\geqslant 37$ weeks gestation. The study shows that G6PD activity is higher in preterm infants than term and near term neonates, but that this increase is limited to the 29-32 week gestational age range. Surprisingly, premature infants born before as well as subsequent to this range had G6PD activity similar to term neonates. The higher G6PD activity in preterm neonates did not affect diagnosis of G6PD deficiency.

effect contributing in part to the control of red blood cell G6PD activity in premature infants.

\section{Authors' affiliations}

O Mesner, D Bader, Department of Neonatology, Bnai Zion Medical Center, Haifa and The Bruce Rappaport Faculty of Medicine, Technion Science Institute, Haifa, Israel

C Hammerman, D Goldschmidt, M Kaplan, Department of Neonatology, Shaare Zedek Medical Center, Jerusalem, Israel B Rudensky, Clinical Hematology Laboratory, Shaare Zedek Medical Center, Jerusalem

C Hammerman, Faculty of Health Sciences, Ben Gurion University of the Negev, Be'er Sheva, Israel

M Kaplan, The Faculty of Medicine of the Hebrew University, Jerusalem

\section{REFERENCES}

1 Beutler E. G6PD deficiency. Blood 1994;84:3613-16.

2 WHO Working Group. Glucose-6-phosphate dehydrogenase deficiency. Bull WHO 1989;67:601-11

3 Oppenheim A, Jury CL, Rund D, et al. G6PD Mediterranean accounts for the high prevalence of G6PD deficiency in Kurdish Jews. Hum Genet 1993;91:293-4.

4 Kaplan M, Renbaum P, Levy-Lahad E, et al. Gilbert Syndrome and glucose-6phosphate dehydrogenase deficiency: a dose-dependent genetic interaction crucial to neonatal hyperbilirubinemia. Proc Natl Acad Sci USA 1997;94:12128-32.

5 Valaes T. Severe neonatal jaundice associated with glucose-6-phosphate dehydrogenase deficiency: pathogenesis and global epidemiology. Acta Paediatr 1994;394(suppl):58-76.

6 Kaplan M, Hammerman C. Severe neonatal hyperbilirubinemia: a potential complication of glucose-6-phosphate dehydrogenase deficiency. Clin Perinatol 1998;25:575-90.

7 Johnson LH, Bhutani VK, Brown AK. System-based approach to management of neonatal jaundice and prevention of kernicterus. J Pediatr 2002; 140:396-403.

8 Travis SF, Kumar SP, Paez PC, et al. Red cell metabolic alterations in postnatal life in term infants: glycolytic enzymes and glucose-6-phosphate dehydrogenase. Pediatr Res 1980;14:1349-52.

9 Konrad PM, Valentine WM, Paglia DE. Enzymatic activities and glutathione content of erythrocytes in the newborn: comparison with red cells of older normal subjects and those with comparable reticulocytosis. Acta Haematol 1972;48:193-201.

10 Kaplan M, Leiter C, Hammerman C, et al. Enzymatic activity in glucose-6phosphate dehydrogenase-normal and -deficient neonates measured with a commercial kit. Clin Chem 1995:41:1665-7.

11 Ciulla AP, Kaster JM, Tetlow AL. Determination of glucose-6-phosphate dehydrogenase deficiency: comparison of three methods. Lab Med 1983; 14:299-302.

12 Marks PA, Gross RT. Erythrocyte glucose-6-phosphate dehydrogenase deficiency: evidence of differences between negroes and Caucasians with respect to this genetically determined trait. J Clin Invest 1959;38:2253-62.

13 Stewart AG, Birnbeck JA. The activities of lactate dehydrogenase, transaminase, and glucose-6-phosphate dehydrogenase in the erythrocytes and plasma of newborn infants. J Pediatr 1962;61:395-404.

14 Herz F, Kaplan E, Scheye ES. A longitudinal study of red cell enzymes in infants of low birth weight. Z Kinderheilk 1975;120:217-21

15 Mohrenweiser HW, Fielek S, Wurzinger KH. Characteristics of enzymes of erythrocytes from newborn infants and adults: activity, thermostability, and elec trophoretic profile as a function of cell age. Am J Hematol 1981;11:125-36.

16 Beutler E, West C, Blume K-G. The removal of leukocytes and platelets from whole blood. J Lab Clin Med 1976;88:328-33.

17 Oski FA, Smith C, Brigandi E. Red cell metabolism in the premature infant. Apparent inappropriate glucose consumption for cell age. Pediatrics 1968;41:473-82. 